\title{
Triticum vulgare para el tratamiento del síndrome genitourinario de la menopausia, Armenia, Colombia
}

\section{Triticum vulgare for the treatment of genitourinary syndrome of menopause, Armenia, Colombia}

\author{
Franklin J. Espitia-De La Hoz* \\ Hathor, Clínica Sexológica, Eje Cafetero, Colombia
}

\section{Resumen}

Objetivo: Evaluar la eficacia y la seguridad de Triticum vulgare en el tratamiento del síndrome genitourinario de la menopausia (SGUM). Método: Estudio cuasiexperimental (antes-después, con grupo control) en mujeres posmenopáusicas (amenorrea $\geq 36$ meses, hormona estimulante del folículo > $40 \mathrm{U} / \mathrm{l}$ y estradiol < $25 \mathrm{pg} / \mathrm{ml}$ ), sexualmente activas, con un índice de maduración vaginal $(I M V)<50$ y pH $\geq 5$, citología cervical negativa (Papanicolaou) y diagnóstico de SGUM, atendidas en el programa de climaterio y menopausia de una clínica privada de mediana complejidad, en Armenia, Quindío (Colombia). Se seleccionaron 207 mujeres con edad promedio de 55,19 \pm 7,28 años. Se realizó un muestreo consecutivo. Se asignaron dos grupos: $A(n=105)$, que recibió $T$. vulgare, y $B(n=102)$, que recibió placebo. Se hizo seguimiento al inicio (basal) y 4,8 y 12 semanas después, utilizando el IMV y el Índice de Función Sexual Femenina (IFSF). Los síntomas del SGUM se evaluaron con una escala visual analógica (EVA). Se aplicó estadística descriptiva. Resultados: La puntuación media del IMV fue mayor en las semanas 4, 8 y 12 en todas las mujeres del grupo $A(p=0,01)$. Se observó una diferencia significativa en el promedio final de la puntuación del IMV de T. vulgare frente al placebo $(p<0,05)$. Al final del estudio, el grupo A mostró una mejoría significativa en la puntuación promedio del IFSF, en comparación con el grupo $B(p<0,001)$. Las puntuaciones de la EVA presentaron una disminución progresiva a lo largo del estudio, pero fueron comparables entre los dos grupos ( $p=0,813)$. Conclusiones: $T$. vulgare es una efectiva, segura e innovadora alternativa, no hormonal, para el tratamiento del SGUM. No se registraron eventos adversos, por lo que se demostró su seguridad.

Palabras clave: Atrofia. Dispareunia. Menopausia. Prurito vulvar. Salud sexual. Vagina.

\section{Abstract}

Objective: To evaluate the efficacy and safety of Triticum vulgare in the treatment of genitourinary syndrome of menopause. Method: Quasi-experimental study (before-after, with control group) in postmenopausal women (amenorrhea $\geq 36$ months, FSH $>40 \mathrm{U} / \mathrm{L}$ and estradiol $<25 \mathrm{pg} / \mathrm{ml}$ ), sexually active, with a vaginal maturation index $(\mathrm{VMI})<50$ and $\mathrm{pH} \geq 5$, negative cervical cytology (Papanicolaou) and with a diagnosis of genitourinary syndrome of menopause (SGUM); who were treated in the climacteric and menopause program of a private clinic of medium complexity, in Armenia, Quindío (Colombia). 207 participants were selected, with a mean age of $55.19 \pm 7.28$ years. A consecutive sampling was carried out. Two groups were assigned: $A(n=105)$ with T. vulgare and $B(n=102)$ with placebo. Follow-up was done at baseline (baseline), four, eight and twelve weeks later, using the VMI and the female sexual function index (IFSF). Symptoms of SGUM were evaluated using a visual analog scale (VAS). Descriptive statistics were applied. Results: The mean score of the IMV was higher in weeks

Correspondencia:

*Franklin J. Espitia-De La Hoz E-mail: espitiafranklin@ hotmail.com

Disponible en internet: $27-10-2021$

Fecha de recepción: 19-12-2020

Fecha de aceptación: 11-08-2021

DOI: $10.24875 /$ RECHOG.M21000017
Rev Chil Obstet Ginecol. 2021;86(4):380-389

www.rechog.com 0048-766X / @ 2021 Sociedad Chilena de Obstetricia y Ginecología. Publicado por Permanyer. Éste es un artículo open access bajo la licencia CC BY-NC-ND (https://creativecommons.org/licenses/by-nc-nd/4.0/). 
4, 8 and 12 in all the participants of group $A(p=0.01)$. A significant difference was observed in the final mean MVI score of T. vulgare versus placebo $(p<0.05)$. At the end of the study, group A showed a significant improvement in the mean IFSF score, compared to placebo $(p<0.001)$. The VAS scores showed a progressive decrease throughout the study but were comparable between the two groups $(p=0.813)$. Conclusions: T. vulgare is an effective, safe and innovative non-hormonal alternative for the treatment of SGUM. No adverse events were recorded, guaranteeing their safety.

Key words: Atrophy. Dyspareunia. Menopause. Pruritus vulvae. Sexual health. Vagina.

\section{Introducción}

El síndrome genitourinario de la menopausia (SGUM) afecta a todos los tejidos del aparato genitourinario, debido a la falta de estrógenos, durante el periodo de la perimenopausia y la posmenopausia'. Se manifiesta en aproximadamente el $15 \%$ de las mujeres premenopáusicas $^{2}$ y el $40-54 \%$ de las posmenopáusicas ${ }^{3}$.

Las mujeres afectadas por SGUM pueden presentar muchos síntomas, siendo los más frecuentes la sequedad vaginal y la dispareunia ${ }^{1-3}$. Las manifestaciones clínicas se dividen, principalmente, en signos y síntomas genitales (externos e internos), urológicos y sexuales (Tabla 1). La citología vaginal muestra un aumento de las células parabasales e intermedias, mientras que las células superficiales disminuyen o están ausentes ${ }^{4}$. A su vez, las mujeres tienen menos probabilidades de tener una flora bacteriana vaginal dominante de Lactobacillus, así como de mantener un $\mathrm{pH}$ vaginal bajo $(2,5-4,5)^{5,6}$.

El diagnóstico se establece con la presencia de al menos dos síntomas, o un signo y un síntoma, que sean considerados como molestos por la paciente, pero que se deban a la menopausia y no a otra causa ${ }^{1,7}$; por lo tanto, es obligatorio realizar una historia clínica completa en toda mujer en quien se sospeche un SGUM.

El abordaje terapéutico del SGUM varía debido a la gravedad de la sintomatología. En presencia de síntomas vaginales leves o moderados, se consideran las terapias no hormonales (hidratantes y lubricantes) como primera línea de manejo, o cuando los estrógenos locales no están indicados o la mujer no puede 0 no desea utilizarlos ${ }^{8}$. En mujeres con síntomas moderados a intensos, los estrógenos locales son la opción de tratamiento más exitosa -en términos de aumentar el índice de maduración vaginal (IMV)-, en pacientes que no presentan síntomas vasomotores y que no mejoran con hidratantes o lubricantes $, 9,10$.

Triticum vulgare es una fitoestimulina que se produce al germinar semillas de gramíneas (en este caso es un extracto obtenido del trigo) y que tiene la capacidad de actuar sobre los tejidos afectados, favoreciendo la reparación mediante la estimulación, la formación, la maduración y la migración de fibroblastos; de esta forma, se genera una síntesis temprana de tejido de granulación, facilitando las óptimas condiciones para que ocurran los procesos secuenciales que conducen a la reepitelización, ya que tiene un efecto mitogénico sobre los fibroblastos ${ }^{11-13}$.

T. vulgare estimula la síntesis de ARNm y de ADN en fibroblastos y linfocitos (con una capacidad similar a la del factor de crecimiento de fibroblastos), activando los fenómenos de cicatrización y reepitelización, al incrementar la quimiotaxis, la movilidad y la maduración de los fibroblastos; todo ello permite la capacidad de síntesis de fibras colágenas y glucosaminoglucanos, aumentando el índice fibroblástico ${ }^{11,14}$, propiedades que aceleran la reparación de los tejidos. En la actualidad se usa comúnmente para el tratamiento de úlceras por decúbito, quemaduras, retrasos en la cicatrización, enfermedades distróficas y en condiciones en las que es necesario estimular la reepitelización o procesos de regeneración tisular ${ }^{15}$.

El IMV y el pH vaginal se evalúan sistemáticamente en los ensayos clínicos, pero no son esenciales en la práctica clínica para hacer el diagnóstico de $\mathrm{SGUM}^{16}$; sin embargo, al evaluar la eficacia y la seguridad de un tratamiento, es de utilidad tanto el análisis citológico como la evaluación del pH vaginal'.

Al conocer que el porcentaje de mujeres posmenopáusicas con atrofia vulvovaginal confirmada por exploración física oscila entre el $67 \%$ y el $98 \%$, mientras que la prevalencia informada por las mujeres sintomáticas es de aproximadamente el $50 \%{ }^{17}$, ante la insuficiente cantidad de investigaciones sobre tratamientos alternativos o complementarios, no hormonales, reconocemos la existencia de una clara necesidad de realizar estudios al respecto ${ }^{15}$. Por ello, este trabajo tuvo como objetivo evaluar la eficacia y la seguridad de T. vulgare, comparado con placebo, en el tratamiento del SGUM.

\section{Método}

\section{Diseño y población}

Se realizó un estudio cuasiexperimental, de medidas repetidas antes y después, con grupo control, sin asignación aleatoria, en mujeres posmenopáusicas 
Rev Chil Obstet Ginecol. 2021;86(4)

Tabla 1. Principales manifestaciones clínicas del síndrome genitourinario de la menopausia

\begin{tabular}{|l|l}
\hline Vulva & $\begin{array}{l}\text { Acortamiento del prepucio y exposición excesiva } \\
\text { del clítoris }\end{array}$ \\
Adelgazamiento y encanecimiento del vello púbico \\
Pérdida de la almohadilla adiposa labial \\
\hline Pérdida del vello púbico
\end{tabular}

Retracción y pérdida de la definición de los labios mayores y menores

Susceptibilidad a irritantes químicos y físicos, a traumas mecánicos e infecciones

Acortamiento, fibrosis, obliteración de la cúpula vaginal o estrechamiento del introito vaginal

Alisamiento de los fondos de saco, aplanamiento de los pliegues/rugosidades vaginales

Disminución de la sensibilidad

Dolor perineal

Vagina Aumento del $\mathrm{pH}$ vaginal $(>5,0)$

Defectos en la mucosa, incluyendo petequias, microfisuras, ulceración e inflamación

Irritación, ardor o picazón

Pérdida de elasticidad

Sequedad vaginal

Susceptibilidad a traumas mecánicos

Vejiga y Disminución de la capacidad vesical

uretra

Incontinencia urinaria de esfuerzo o de urgencia

Infecciones urinarias recurrentes

Meato uretral prominente (carúnculas uretrales)

Prolapso uretral

Síntomas como frecuencia, disuria, nicturia y urgencia

Sexuales Disminución de la lubricación

Disorgasmia

Dispareunia

Sangrado poscoital

Trastorno de la excitación o del deseo

(amenorrea $\geq 36$ meses, hormona estimulante del folículo $>40 \mathrm{U} / \mathrm{l}$ y estradiol $<25 \mathrm{pg} / \mathrm{ml}$ ), sexualmente activas, con un IMV $<50$ y $\mathrm{pH} \geq 5$, citología cervical negativa (Papanicolaou) y diagnóstico de SGUM. Se atendieron en el programa de climaterio y menopausia de una clínica privada de mediana complejidad, que atiende población perteneciente al régimen contributivo y subsidiado por el Estado en el Sistema General de Seguridad Social, en Armenia, Quindío (Colombia), desde el 1 de mayo hasta el 30 de noviembre de 2019. Se excluyeron mujeres con histerectomía, con antecedente de cáncer de endometrio o mama, sangrado vaginal anormal, antecedente de disfunción sexual, alergia a $T$. vulgare, antecedente de cirugía pélvica reciente ( $\leq 3$ meses), imagen ecográfica con endometrio $\geq 4 \mathrm{~mm}$, cirugía pélvica previa con malla, ooforectomía, infección genital activa, que hubieran recibido terapia hormonal en los 3 meses previos al estudio, con datos incompletos en la historia clínica y las que no desearon participar.

El tamaño de muestra calculado fue de 100 participantes en cada grupo. Se calculó el tamaño de muestra para la comparación de medias de puntuación del IMV (test bilateral, nivel de confianza del $95 \%$, poder estadístico del $90 \%$, precisión 0,5 y varianza 1), esperando unas pérdidas del 15\%. Se asignaron 105 participantes al grupo de $T$. vulgare (grupo A) y 102 al de placebo (grupo B).

\section{Procedimiento}

El investigador principal detectó a las pacientes en la consulta de climaterio y menopausia, y se les asignó el código «N95.2 Vaginitis atrófica posmenopáusica» de la Clasificación Internacional de Enfermedades (10. ${ }^{\text {a }}$ revisión).

Se tomó una muestra de flujo vaginal a todas las participantes para determinar la puntuación del IMV $<50$ y el $\mathrm{pH} \geq 5$. Si la mujer cumplía con los criterios, se le informaba de los objetivos del estudio y se le solicitaba la firma del consentimiento informado. Una vez obtenido el consentimiento, una auxiliar de enfermería, encargada de la recolección del instrumento estandarizado, le daba instrucciones para el autodiligenciamiento del cuestionario del Índice de Función Sexual Femenina (IFSF), de manera individual y en un ambiente privado.

EI IMV expresa la proporción de los tipos celulares vaginales en tres cantidades (mediante una citología), de 100 células contadas en el frotis. Se clasifica el porcentaje de células basales/parabasales, intermedias y superficiales, y de esta forma se calcula el IMV. En las mujeres con baja concentración de estrógenos, el porcentaje de células parabasales e intermedias presenta un aumento significativo en comparación con las mujeres premenopáusicas ${ }^{18-20}$.

EI IMV se cuantifica mediante la proporción de células vaginales parabasales, intermedias y superficiales, 
y se calcula el valor estrogénico $[0,2 \times(\%$ parabasal $)$ $+0,6 \times$ (\% intermedio) $+1,0 \times$ (\% superficial)] (rango 20-100). Un resultado de 0-49 se considera un efecto estrogénico ausente o bajo, 50-64 como moderado y 65-100 como alto ${ }^{19,20}$.

El IFSF es un instrumento de encuesta validado que consta de 19 ítems. Ha sido diseñado para evaluar la función sexual femenina durante las 4 semanas anteriores ${ }^{21}$. Evalúa seis dominios: deseo, excitación, lubricación, orgasmo, satisfacción y dolor. Cada dominio se puntúa mediante una escala Likert de 0 a 50 de 1 a 5 , con una puntuación total que va de 2 a 36; con un punto corte de 26,55 existe el riesgo de disfunción sexual, o cuando la puntuación de algún dominio es menor de 3,6 puntos p $^{22,23}$.

Se obtuvo la información de las características sociodemográficas y clínicas de la historia clínica. La extracción de datos la realizó un médico general (que apoya al investigador principal en las actividades administrativas) en un formato para recolectar las variables descritas. Luego los datos fueron ingresados en una base de datos protegida por un revisor, quien se encargó de su digitalización y verificación en Excel de Office $®$, con el fin de poder detectar datos faltantes o fuera de rango. La información posteriormente fue revisada por una enfermera profesional (especialista en auditoría en salud) y comparada con el formato de extracción de datos, con el propósito de incluir los faltantes.

Las participantes, en su totalidad, fueron atendidas por el investigador principal. A las mujeres se les realizaba una citología cervicovaginal (Papanicolau) y ultrasonografía pélvica transvaginal antes del inicio de la terapia, para evaluar el tamaño uterino, el grosor endometrial y la anatomía anexial.

\section{Intervención}

Una enfermera, integrante del grupo de investigación, dividió a las participantes en dos grupos: grupo A $(n=105)$, que recibieron $5 \mathrm{~g}$ diarios (crema vaginal al $20 \%$, equivalentes a $1 \mathrm{~g}$ de extracto de $T$. vulgare) y grupo $B(n=102)$, que recibieron $5 \mathrm{~g}$ diarios de placebo (un lubricante a base de agua). El fármaco de estudio y el placebo tenían idénticas características (apariencia, olor y textura). La forma de incluir a las mujeres en cada grupo fue mediante asignación de números pares e impares del documento de identidad, correspondiendo los pares al grupo A y los impares al grupo B. Las participantes de ambos grupos usaron la medicación a diario, en la noche, al acostarse, durante 12 semanas. Ambos tratamientos se administraron con un aplicador intravaginal. Las participantes fueron evaluadas al inicio del estudio (basal) y a las 4,8 y 12 semanas después del inicio del tratamiento.

En cada control se evaluaron el IMV a partir de dos muestras de citología (extraídas de la parte inferior del saco vaginal derecho e izquierdo), el pH vaginal (medido en la secreción vaginal utilizando una tira reactiva), el IFSF y los síntomas del SGUM (evaluados con una escala visual analógica de $10 \mathrm{~cm}$ de longitud, donde 0 es ausencia total del síntoma y 10 es el grado máximo del síntoma).

Al término del periodo del estudio se expresó un juicio global de tolerabilidad y de eficacia terapéutica, por separado, tanto por la paciente como por el investigador, de acuerdo con la siguiente escala de valoración: ausencia de síntomas vaginales (sequedad vaginal, dispareunia y prurito) por parte de la paciente y por parte del investigador, y ausencia de signos (pérdida de rugosidad del epitelio vaginal, fragilidad y sequedad de la mucosa). La evaluación se hizo con una escala porcentual: $\geq 75 \%$, excelente (ausencia del signo o síntoma); $\geq 51 \%$ y $\leq 74 \%$, buena (signos o síntomas leves); $\geq 26 \%$ y $\leq 50 \%$, regular (signos o síntomas moderados); $y \leq 25 \%$, mala (el signo o síntoma se considera muy irritante y grave).

El cumplimiento del tratamiento se evaluó contando y registrando el número de aplicadores no utilizados. La ocurrencia de eventos adversos se evaluó como parámetros de seguridad. Se garantizó el doble ciego, ya que tanto las participantes como el investigador desconocían a qué grupo pertenecían.

\section{Análisis estadístico}

Se realizó un análisis descriptivo, con frecuencias absolutas y relativas, y porcentajes para variables nominales; media y desviación estándar para las medidas de tendencia central y de dispersión. Se compararon los resultados obtenidos en los dos grupos, así como entre la medición inicial y la medición posprueba, mediante las pruebas de ji al cuadrado para los datos cualitativos y $t$ de Student para los datos cuantitativos. Se consideraron estadísticamente significativos los valores de $p<$ 0,05 . El análisis de los datos se realizó con el paquete estadístico SPSS, versión 21, para Windows.

\section{Aspectos éticos}

El presente estudio fue aprobado por el Comité de Ética Médica y cumplió los principios éticos de investigaciones médica en seres humanos establecidos en la Declaración de Helsinki ${ }^{24}$ y en la Resolución 8430 
de 1993 del Ministerio de Salud de Colombia, por la cual se establecen las normas científicas, técnicas y administrativas para investigación en salud ${ }^{25}$. Se solicitó la firma del consentimiento informado y se garantizó la total confidencialidad de los datos.

\section{Variables analizadas}

Se analizaron variables sociodemográficas (edad, nivel educativo, zona de residencia, nivel socioeconómico y régimen de afiliación al Sistema General de Seguridad Social en Colombia), comorbilidad, índice de masa corporal (IMC), gestaciones y paridad, y sintomatología de SGUM. Se indagó la edad de inicio de la actividad sexual, la frecuencia de relaciones sexuales, el número de parejas sexuales y el tipo de relación sexual. Se interrogaron, además, los dominios de la encuesta IFSF. Adicionalmente, se evaluó el estatus hormonal celular vaginal mediante el IMV, el pH vaginal y los síntomas de SGUM mediante una escala visual analógica.

\section{Resultados}

De las 752 mujeres elegibles, 438 no cumplieron con los criterios de inclusión, por lo que quedaron 314 como población de estudio. De estas, 76 (27,27\%) tenían datos incompletos y 31 no aceptaron participar. Finalmente, se reclutaron 207 mujeres con diagnóstico de SGUM atendidas en el periodo del estudio. No hubo pérdida de participantes a lo largo del seguimiento.

De las 207 mujeres incluidas en la investigación, el $80,67 \%$ residían en el área urbana, el $74,87 \%$ eran amas de casa, el $58,45 \%$ hispanas, el $54,58 \%$ de estrato socioeconómico medio y el $46,37 \%$ estaban casadas o eran convivientes. El $47,82 \%$ eran mayores de 55 años. En relación con la escolaridad, el 65,21\% había completado la secundaria y el resto tenían un nivel superior de escolaridad. El $89,37 \%$ profesaban la religión católica. El $87,92 \%$ se encontraban afiliadas al régimen contributivo de salud. El análisis de los datos no mostró diferencias significativas entre los dos grupos con respecto a las variables evaluadas $(p>0,05)$.

La edad media de las participantes que recibieron T. vulgare fue de $54,6 \pm 7,14$ años (límites: 42 y 78), y la de aquellas que recibieron placebo fue de 55,8 \pm 8,34 años (límites: 42 y 75), sin diferencia estadísticamente significativa $(p=0,081)$. La edad promedio del grupo total de participantes fue de $55,19 \pm 7,28$ años. La edad media de las parejas fue de 61,59 \pm 7,61 años (límites: 45 y 78).
En los antecedentes patológicos, el $24,63 \%$ de las participantes presentaba comorbilidad, siendo la hipertensión arterial la más frecuente (Tabla 1).

El IMC en la población total fue de $25,17 \pm 6,82$. El $59,42 \%$ presentaron un IMC por encima de lo normal, el $36,23 \%$ sobrepeso y el $23,18 \%$ obesidad.

El $89,55 \%$ de las mujeres habían estado embarazadas por lo menos en una ocasión; el 69,56\% habían tenido parto vaginal y el $30,43 \%$ parto por cesárea; y el 27,53\% habían presentado por lo menos un aborto.

En relación con el comportamiento sexual, se encontró que el 86,95\% inició su vida sexual a los 16 años. Las relaciones sexuales eran predominantemente vaginales y orales $(90,82 \%)$. La frecuencia con que tenían actividad sexual reportó una mediana de tres veces al mes (rango: 0-5).

El origen de la menopausia, en la mayoría de las mujeres $(81,15 \%)$ fue fisiológico.

Las pacientes fueron completamente adherentes al tratamiento, ya que completaron las 12 semanas con un cumplimiento del $97,1 \%$ en ambos grupos $(96,19 \%$ para T. vulgare y $98,03 \%$ para el placebo); por lo tanto, todas se incluyeron en el análisis de la población.

El síntoma más frecuente fue la sequedad vaginal, mientras que el signo más común fue la pérdida de rugosidad del epitelio vaginal. El $36,23 \%$ presentaron tres o más síntomas. En la tabla 2 se describen los signos y síntomas descritos por las pacientes y encontrados durante la exploración física, antes y después de recibir el tratamiento.

En las mujeres que recibieron $T$. vulgare, la puntuación media del IMV aumentó de forma significativa en la semana 12 en comparación con el inicio $(p<0,05)$. El IMV indicó un incremento en las células superficiales del $2,79 \%$ al $37,8 \%$ y en las células intermedias del $35,4 \%$ al $69,6 \%$, mientras que el porcentaje de células parabasales disminuyó del $56,7 \%$ al $6,24 \%$ (Tabla 3).

Los puntajes generales del IMV aumentaron significativamente en ambos grupos durante el curso del estudio ( $p<0,0001)$. No obstante, al comparar en cada grupo los cambios encontrados entre el inicio y el final de la intervención, en el grupo A se observó una disminución significativa en el porcentaje de células parabasales, así como un significativo incremento en el porcentaje de células intermedias y superficiales $(p<0,0001)$ (Tabla 3).

La atenuación en la puntuación de la escala visual analógica después de 8 semanas fue estadísticamente significativa en ambos grupos $(p<0,001)$; la diferencia de atenuación entre los grupos a lo largo del seguimiento no fue estadísticamente significativa $(p=0,702)$ 
Tabla 2. Sintomatología del síndrome genitourinario de la menopausia antes y después de la intervención

\begin{tabular}{|c|c|c|c|c|c|c|}
\hline \multirow[t]{2}{*}{ Sintomatología } & \multicolumn{2}{|c|}{$\begin{array}{l}\text { Grupo Triticum vulgare } \\
\qquad(\mathrm{n}=105)\end{array}$} & \multirow[t]{2}{*}{ p } & \multicolumn{2}{|c|}{$\begin{array}{l}\text { Grupo placebo } \\
\qquad(\mathrm{n}=102)\end{array}$} & \multirow[t]{2}{*}{ p } \\
\hline & Antes & Después & & Antes & Después & \\
\hline Carúnculas uretrales proliferativas & $5,71 \%$ & $5,71 \%$ & NS & $3,92 \%$ & $3,92 \%$ & NS \\
\hline Disminución de la lubricación vaginal & $68,57 \%$ & $29,52 \%$ & 0,001 & $65,68 \%$ & $50,98 \%$ & 0,189 \\
\hline $\begin{array}{l}\text { Disminución del grosor de los labios } \\
\text { mayores }\end{array}$ & $42,85 \%$ & $42,85 \%$ & NS & $43,13 \%$ & $43,13 \%$ & NS \\
\hline Dispareunia & $71,42 \%$ & $34,28 \%$ & 0,001 & $72,54 \%$ & $63,72 \%$ & 0,213 \\
\hline Infecciones urinarias de repetición & $27,61 \%$ & $27,61 \%$ & NS & $25,49 \%$ & $25,49 \%$ & NS \\
\hline Irritación vaginal & $23,8 \%$ & $6,66 \%$ & 0,001 & $25,49 \%$ & $10,78 \%$ & 0,012 \\
\hline Meato uretral prominente & $14,28 \%$ & $14,28 \%$ & NS & $15,68 \%$ & $15,68 \%$ & NS \\
\hline $\begin{array}{l}\text { Pérdida de los pliegues y rugosidades } \\
\text { vaginales }\end{array}$ & $56,19 \%$ & $56,19 \%$ & NS & $57,84 \%$ & $57,84 \%$ & NS \\
\hline Prurito (ardor, quemazón) genital & $12,38 \%$ & $2,85 \%$ & 0,001 & $13,72 \%$ & $8,82 \%$ & 0,153 \\
\hline Sequedad vaginal & $80,95 \%$ & $12,38 \%$ & 0,001 & $79,41 \%$ & $41,17 \%$ & 0,042 \\
\hline Síntomas urinarios & $32,38 \%$ & $18,09 \%$ & 0,001 & $30,39 \%$ & $26,47 \%$ & 0,081 \\
\hline Sinusorragia & $9,52 \%$ & $5,71 \%$ & 0,045 & $8,82 \%$ & $6,86 \%$ & 0,108 \\
\hline
\end{tabular}

NS: no significativo.

(Tabla 3). Al considerar la diferencia media en la puntuación general de cada grupo en la cuarta, octava y duodécima semana no se observaron diferencias significativas entre los grupos ( $p>0,05$ ).

En ambos grupos, las puntuaciones medias para los dominios del IFSF aumentaron de forma significativa en la semana 12 , en comparación con el inicio $(p<0,05)$ (Tabla 4).

La puntuación total en el IFSF tuvo un incremento estadísticamente significativo en los dos grupos desde las 4 semanas (diferencias que se mantuvieron a las 8 y 12 semanas), observándose un decremento significativo en el dominio dolor $(p<0,05)$ (Tabla 4). Al final del estudio, en el grupo A se observó una diferencia significativa en el promedio de la puntuación final del IFSF frente a la puntuación del grupo que recibió placebo $(27,94 \pm 6,9$ frente a $24,29 \pm 8,4 ; p<0,05)$.

El grupo con placebo reportó una mediana de 2 (rango: 0-4) en la frecuencia mensual de coito, frente a 5 (rango: $0-7)$ en el grupo con T. vulgare $(p<0,001)$.

En la evaluación de la sintomatología más frecuente antes de iniciar el tratamiento se evidenció que ambos grupos presentaban una alta prevalencia de sequedad vaginal: el $80,95 \%$ en el grupo con $T$. vulgare (intervalo de confianza del $95 \%$ [IC95\%]: $78,59-82,07$ ) y el $79,41 \%$ en el grupo con placebo (IC95\%: 78,59-82,07), la cual fue similar en ambos grupos $(p>0,05)$. Luego de 12 semanas de tratamiento, el grupo que recibió $T$. vulgare presentó una disminución del $68,57 \%$, mientras que el grupo que recibió placebo tuvo una disminución del $38,23 \%$, diferencia que fue significativa (odds ratio [OR]: 30,34; IC95\%: 11,13-32,67; $p=0,001$ ).

La frecuencia de la disminución de la lubricación vaginal al iniciar el seguimiento fue similar en ambos grupos: $68,57 \%$ en el grupo con T. vulgare (IC95\%: $65,72-71,48)$ y $65,68 \%$ en el grupo con placebo (IC95\%: $64,28-67,35 ; p>0,05)$. A las 12 semanas de la intervención, el grupo que recibió $T$. vulgare presentó una disminución del 39,04\%, mientras que en el grupo con placebo la disminución fue del 14,07\%; este resultado se traduce en una reducción significativa (OR: 24,97; IC95\%: 13,25-36,48; $p=0,001$ ).

Al final de la intervención, en el grupo placebo, la dispareunia persistía elevada (63,72\%; IC95\%: 54,62$65,87)$, mientras que en el grupo de $T$. vulgare experimentó un claro descenso (34,28\%; IC95\%: 13,86-45,27), con diferencias significativas entre las determinaciones previa y posterior a la intervención del grupo con placebo (37,64; IC95\%: 21,87-42,51). 
Tabla 3. Evaluación de los resultados de los cambios en el índice de maduración vaginal y la escala visual analógica en los dos grupos

\begin{tabular}{|c|c|c|c|c|c|c|}
\hline & Grupos & Inicio & 4 semanas & 8 semanas & 12 semanas & $\stackrel{p}{\text { (inicio vs. final) }}$ \\
\hline \multicolumn{7}{|c|}{ IMV (\% células) } \\
\hline \multirow[t]{3}{*}{ Parabasales } & T. vulgare & $79,5 \pm 35,1$ & $53,8 \pm 37,6$ & $26,9 \pm 42,8$ & $21,7 \pm 35,2$ & 0,001 \\
\hline & Placebo & $78,1 \pm 36,2$ & $69,5 \pm 38,4$ & $65,9 \pm 41,7$ & $55,9 \pm 37,8$ & 0,048 \\
\hline & Comparación & NS & 0,045 & 0,027 & 0,009 & \\
\hline \multirow[t]{3}{*}{ Intermedias } & T. vulgare & $14,3 \pm 17,7$ & $22,9 \pm 19,4$ & $37,4 \pm 23,8$ & $44,5 \pm 17,9$ & 0,001 \\
\hline & Placebo & $15,3 \pm 16,9$ & $23,8 \pm 19,1$ & $26,7 \pm 24,3$ & $29,5 \pm 18,2$ & 0,033 \\
\hline & Comparación & NS & NS & 0,042 & 0,036 & \\
\hline \multirow[t]{3}{*}{ Superficiales } & T. vulgare & $6,2 \pm 13,5$ & $23,3 \pm 17,8$ & $35,7 \pm 21,7$ & $33,8 \pm 25,6$ & 0,001 \\
\hline & Placebo & $6,6 \pm 14,1$ & $7,6 \pm 17,7$ & $7,4 \pm 18,9$ & $14,6 \pm 15,7$ & 0,001 \\
\hline & Comparación & NS & 0,036 & 0,003 & 0,003 & \\
\hline \multicolumn{7}{|l|}{ EVA (cm) } \\
\hline \multirow[t]{3}{*}{ Sequedad vaginal } & T. vulgare & $7,65 \pm 2,1$ & $6,29 \pm 1,7$ & $5,17 \pm 1,4$ & $3,34 \pm 1,5$ & 0,001 \\
\hline & Placebo & $7,81 \pm 2,7$ & $6,17 \pm 1,8$ & $5,09 \pm 1,4$ & $3,29 \pm 1,2$ & 0,001 \\
\hline & Comparación & NS & NS & NS & NS & \\
\hline \multirow[t]{3}{*}{ Dispareunia } & T. vulgare & $8,49 \pm 2,4$ & $6,82 \pm 1,9$ & $4,91 \pm 1,5$ & $3,57 \pm 1,8$ & 0,001 \\
\hline & Placebo & $8,52 \pm 2,1$ & $6,41 \pm 1,5$ & $4,87 \pm 1,2$ & $3,54 \pm 1,5$ & 0,001 \\
\hline & Comparación & NS & NS & NS & NS & \\
\hline \multirow[t]{3}{*}{ Lubricación } & T. vulgare & $7,38 \pm 2,7$ & $6,41 \pm 1,5$ & $4,75 \pm 1,3$ & $3,29 \pm 1,6$ & 0,001 \\
\hline & Placebo & $7,29 \pm 2,4$ & $6,03 \pm 1,8$ & $4,61 \pm 1,5$ & $3,18 \pm 1,2$ & 0,001 \\
\hline & Comparación & NS & NS & NS & NS & \\
\hline \multirow[t]{3}{*}{$\mathrm{pH}$} & T. vulgare & $6,31 \pm 1,3$ & $4,62 \pm 1,1$ & $4,27 \pm 1,5$ & $3,91 \pm 1,8$ & 0,001 \\
\hline & Placebo & $6,27 \pm 1,8$ & $5,71 \pm 1,2$ & $5,19 \pm 1,8$ & $4,95 \pm 1,5$ & 0,001 \\
\hline & Comparación & NS & 0,036 & 0,042 & 0,039 & \\
\hline
\end{tabular}

EVA: escala visual analógica; IMV: índice de maduración vaginal; NS: no significativo.

Finalmente, al terminar el estudio, en el grupo de T. vulgare se observó un descenso de los tres síntomas más frecuentes del SGUM, bajando del 80,95\% (IC95\%: $26,83-83,92)$ al $12,38 \%$ (IC95\%: 11,57-29,81\%; $p<0,05)$, y alcanzando diferencias estadísticamente significativas al comparar ambos grupos en la valoración posintervención $(p<0,05)$.

Se pudo apreciar un descenso continuo del pH en ambos grupos, siendo más acentuado en el grupo de T. vulgare después de 12 semanas; las diferencias fueron significativas entre las medias de los grupos respecto a la valoración posintervención (OR: 1,04; IC95\%: 1,01-1,84; $p<0,05)$.
A lo largo del seguimiento no se reportaron infecciones urogenitales en los grupos estudiados (infección urinaria, vaginosis bacteriana o candidiasis). No se registraron eventos adversos con ninguno de los dos productos; ambos fueron bien tolerados, lo que garantiza su seguridad.

Al final del estudio, en cuanto a la tolerabilidad del tratamiento, el $84,76 \%$ de las mujeres del grupo A calificaron la crema vaginal como excelente, el 7,61\% como buena, el $4,76 \%$ como regular y el 2,85\% como mala; por su parte, en el grupo B, el $59,8 \%$ calificaron el gel vaginal como excelente, el $16,66 \%$ como bueno, el $12,74 \%$ como regular y el $10,78 \%$ como malo; 
Tabla 4. Resultados del seguimiento del Índice de Función Sexual Femenina en los dos grupos

\begin{tabular}{|c|c|c|c|c|c|c|}
\hline & Grupos & Inicio & 4 semanas & 8 semanas & 12 semanas & $\begin{array}{c}p \\
\text { (inicio vs. final) }\end{array}$ \\
\hline \multicolumn{7}{|c|}{ Dominios } \\
\hline \multirow[t]{3}{*}{ Deseo } & T. vulgare & $3,41 \pm 1,3$ & $3,62 \pm 1,9$ & $4,24 \pm 1,5$ & $4,42 \pm 1,1$ & 0,001 \\
\hline & Placebo & $3,52 \pm 1,1$ & $3,57 \pm 1,7$ & $3,65 \pm 1,3$ & $3,78 \pm 1,4$ & 0,001 \\
\hline & Comparación & NS & NS & 0,048 & 0,042 & \\
\hline \multirow[t]{3}{*}{ Excitación } & T. vulgare & $3,53 \pm 1,6$ & $3,97 \pm 1,1$ & $4,81 \pm 1,7$ & $4,93 \pm 1,2$ & 0,001 \\
\hline & Placebo & $3,47 \pm 1,3$ & $3,62 \pm 1,1$ & $3,94 \pm 1,2$ & $3,99 \pm 1,5$ & 0,001 \\
\hline & Comparación & NS & NS & 0,045 & 0,039 & \\
\hline \multirow[t]{3}{*}{ Lubricación } & T. vulgare & $3,38 \pm 1,4$ & $3,85 \pm 1,5$ & $4,76 \pm 1,2$ & $4,85 \pm 1,1$ & 0,001 \\
\hline & Placebo & $3,41 \pm 1,6$ & $3,53 \pm 1,5$ & $3,67 \pm 1,4$ & $3,75 \pm 1,7$ & 0,001 \\
\hline & Comparación & NS & NS & 0,048 & 0,042 & \\
\hline \multirow[t]{3}{*}{ Orgasmo } & T. vulgare & $3,77 \pm 1,2$ & $3,99 \pm 1,6$ & $4,49 \pm 1,3$ & $4,67 \pm 1,2$ & 0,001 \\
\hline & Placebo & $3,79 \pm 1,5$ & $3,91 \pm 1,3$ & $3,98 \pm 1,2$ & $4,07 \pm 1,1$ & 0,001 \\
\hline & Comparación & NS & NS & 0,075 & 0,078 & \\
\hline \multirow[t]{3}{*}{ Satisfacción } & T. vulgare & $3,82 \pm 1,5$ & $4,48 \pm 1,8$ & $4,95 \pm 1,9$ & $4,99 \pm 1,1$ & 0,001 \\
\hline & Placebo & $3,84 \pm 1,7$ & $3,95 \pm 1,4$ & $4,23 \pm 1,1$ & $4,34 \pm 1,2$ & 0,001 \\
\hline & Comparación & NS & 0,081 & 0,075 & 0,084 & \\
\hline \multirow[t]{3}{*}{ Dolor } & T. vulgare & $4,98 \pm 1,7$ & $4,56 \pm 1,2$ & $4,03 \pm 1,8$ & $4,08 \pm 1,2$ & 0,001 \\
\hline & Placebo & $4,95 \pm 1,4$ & $4,48 \pm 1,2$ & $4,39 \pm 1,3$ & $4,36 \pm 1,5$ & 0,001 \\
\hline & Comparación & NS & NS & NS & NS & \\
\hline \multirow[t]{3}{*}{ Total } & T. vulgare & $22,89 \pm 8,7$ & $24,47 \pm 9,1$ & $27,28 \pm 9,4$ & $27,94 \pm 6,9$ & 0,001 \\
\hline & Placebo & $22,98 \pm 8,6$ & $23,06 \pm 8,2$ & $23,86 \pm 7,5$ & $24,29 \pm 8,4$ & 0,001 \\
\hline & Comparación & NS & 0,075 & 0,039 & 0,042 & \\
\hline
\end{tabular}

NS: no significativo.

encontrándose diferencias estadísticamente significativas $(p=0,006)$.

\section{Discusión}

Al finalizar el presente estudio, la puntuación del IMV fue expresivamente diferente en las dos intervenciones; no obstante, después de 4 semanas se observó una diferencia significativa a favor de $T$. vulgare $(p<0,05)$. El éxito del tratamiento fue mayor en el grupo de $T$. vulgare que en el grupo con placebo, y la diferencia se mantuvo a lo largo del estudio; sin embargo, conociendo la capacidad regenerativa, no sorprende que la fitoestimulina diera como resultado una maduración escamosa del epitelio vaginal ${ }^{11,13}$.
En esta investigación se encontró una importante asociación entre el uso de $T$. vulgare y la mejora en la puntuación del IFSF; también se observó una disminución en el dominio dolor, lo cual se asoció con un incremento de la satisfacción. Esto se podría explicar porque T. vulgare determina una marcada aceleración de los procesos de reparación tisular y estimula la quimiotaxis y la maduración fibroblástica, así como el incremento significativo del índice fibroblástico, que son puntos cruciales en los procesos de reparación de los tejidos ${ }^{15,26,27}$; adicionalmente, posee potenciales propiedades antiinflamatorias, siendo de utilidad en el tratamiento de la inflamación vaginal y la distrofia vulvar ${ }^{28}$.

Los resultados de este estudio evidencian que T. vulgare favoreció la frecuencia coital mensual, lo 
cual puede deberse a una mejoría en la excitación y la lubricación, con disminución de la dispareunia y de la sequedad vaginal, ya que el uso de la fitoestimulina favorece la cicatrización al aumentar el número de vasos sanguíneos neoformados ${ }^{29}$.

Se encontró que $T$. vulgare tiene una notable diferencia, frente al placebo, en el tratamiento del SGUM, pues la mayoría de las mujeres alcanzaron resultados satisfactorios desde las 4 semanas de tratamiento, lo cual puede asociarse a que $T$. vulgare es capaz de favorecer la regeneración tisular, la proliferación de fibroblastos y la fase de remodelación ${ }^{12,30}$, que se encuentra alterada en las mujeres con SGUM.

En cuanto a los eventos adversos, no se reportaron en ninguno de los dos grupos, siendo bien tolerados tanto T. vulgare como el placebo (lubricante). Romanelli, et al. ${ }^{31}$, en un estudio piloto aleatorizado, hicieron una evaluación clínica de la eficacia y la seguridad de diversas presentaciones de $T$. vulgare, y demostraron la ausencia de efectos adversos.

EI SGUM es una condición crónica, progresiva e irreversible (si no recibe tratamiento), que tiene un alto impacto negativo en la calidad de vida de la mujer que lo padece; por lo tanto, requiere un manejo constante de la sintomatología ${ }^{1,732}$, y aquí $T$. vulgare ha demostrado su positiva contribución como terapia no hormonal.

Al ser muchas las mujeres reacias a plantear a sus médicos las dificultades que les genera el SGUM, por vergüenza o razones culturales, sumado a que en ocasiones los profesionales de la salud no tocan el tema durante la consulta porque se sienten incómodos al discutir temas sexuales ${ }^{33}$ o porque no tienen las competencias para abordarlos, existe una importante falta de conocimiento sobre la gama de opciones de tratamiento (no hormonales) eficaces y seguras que se encuentran disponibles ${ }^{7,32}$.

Es conveniente una campaña de concientización pública combinada con educación continua para los profesionales de la salud, con el fin de dar a conocer las diferentes alternativas terapéuticas para el tratamiento del SGUM, en la búsqueda de fomentar la pronta y oportuna intervención, para evitar la catastrófica aparición de la constelación de síntomas que lo caracteriza ${ }^{1,32}$.

A la luz de los presentes resultados, T. vulgare es en un preparado de elección en aquellas mujeres con SGUM y constricción vaginal que no quieren usar estrógenos vaginales, o como tratamiento complementario de las diferentes terapias existentes en mujeres con dispareunia; adicionalmente, se puede usar varias veces a la semana, independientemente de la actividad sexual ${ }^{15}$. Sus efectos son más a largo plazo que los de los lubricantes y están destinados a aliviar la sequedad vaginal y la vaginitis atrófica.

Esta es la primera investigación que se realiza con T. vulgare para el tratamiento del SGUM; por eso, ante la falta de estudios previos, no es posible contrastar los resultados obtenidos.

Es importante mencionar que, aunque no se hayan observado eventos adversos en este estudio, eso no significa que no puedan producirse; es posible que el tamaño de muestra sea muy limitado como para poder afirmar la ausencia de eventos adversos.

Una de las limitaciones de esta investigación es que se limitó a un seguimiento de 12 semanas, lo que excluyó la evaluación a largo plazo de los efectos de T. vulgare; adicionalmente, no se ha podido contrastar los resultados con otros estudios, lo que podría haber mejorado el análisis de los hallazgos. Además, al tratarse de una investigación cuasiexperimental, existe la posibilidad de presentar sesgos, lo cual puede comprometer la aplicabilidad de los resultados. Por otro lado, se contó con una muestra reducida de la población afiliada al régimen subsidiado, debido a que la mayor proporción de estas pacientes asisten a centros hospitalarios públicos, por asuntos de contratación.

En cuanto a las fortalezas del estudio, se contó con instrumentos validados y ampliamente conocidos para este tipo de investigaciones, el tamaño de la muestra fue adecuado (a pesar de la baja casuística, es suficiente para compararla contra placebo) y es el primer estudio realizado en Colombia sobre la eficacia de T. vulgare en mujeres con SGUM.

Puesto que los resultados de esta investigación indican que $T$. vulgare es eficaz y seguro, se justifican más estudios (aleatorizados, doble ciegos y controlados con placebo), con un tamaño de muestra más grande, para evaluar los efectos a largo plazo.

\section{Conclusiones}

T. vulgare, según los datos obtenidos, es una efectiva, segura e innovadora alternativa no hormonal para aliviar los síntomas de la atrofia vaginal en mujeres posmenopáusicas, convirtiéndose en otra opción terapéutica especialmente en las mujeres que no pueden o no desean usar la terapia hormonal. Es importante que se sigan haciendo estudios prospectivos con T. vulgare, con evaluaciones objetivas, a fin de comparar sus beneficios frente a las otras diferentes opciones no hormonales. También conviene que los estudios investiguen la eventual capacidad de T. vulgare para restaurar el epitelio vaginal a un estado similar al del estado 
premenopáusico, y si facilita que aumente la cantidad de Lactobacillus.

\section{Agradecimientos}

Agradezco a las mujeres participantes en este estudio, a Hathor, Clínica Sexológica, por facilitar sus instalaciones, al personal de enfermería, a los bioestadísticos y a los epidemiólogos que nos apoyaron. Asimismo, quiero destacar la labor de mi amigo y maestro, el doctor Jaime Gallego Arbeláez, por contagiarme su sed de conocimiento.

\section{Financiamiento}

En este proyecto no se contó con el financiamiento de terceros.

\section{Conflicto de intereses}

\section{El autor declara no tener conflictos de intereses.}

\section{Responsabilidades éticas}

Protección de personas y animales. Los autores declaran que los procedimientos seguidos se conformaron a las normas éticas del comité de experimentación humana responsable y de acuerdo con la Asociación Médica Mundial y la Declaración de Helsinki.

Confidencialidad de los datos. Los autores declaran que han seguido los protocolos de su centro de trabajo sobre la publicación de datos de pacientes.

Derecho a la privacidad y consentimiento informado. Los autores han obtenido el consentimiento informado de los pacientes $y / 0$ sujetos referidos en el artículo. Este documento obra en poder del autor de correspondencia.

\section{Bibliografía}

1. Espitia De la Hoz FJ. Prevalence of genitourinary syndrome of menopause and impact on sexuality of women in Quindío (Colombia), 2013-2016. Rev Colomb Obstet Ginecol. 2018;69:249-59.

2. Palacios S. Managing urogenital atrophy. Maturitas. 2009;63:315-8.

3. DiBonaventura M, Luo X, Moffatt M, Bushmakin AG, Kumar M, Bobula J. The association between vulvovaginal atrophy symptoms and quality of life among postmenopausal women in the United States and Western Europe. J Womens Health (Larchmt). 2015;24:713-22.

4. Bachmann GA, Cheng RJ, Rovner E. Vulvovaginal complaints. En: Lobo RA, editor. Treatment of the postmenopausal woman: basic and clinical aspects. $3^{\text {rd }}$ ed. Burlington, MA: Academic Press; 2007. p. 263-70.

5. Brotman RM, Shardell MD, Gajer P, Fadrosh D, Chang K, Silver MI, et al. Association between the vaginal microbiota, menopause status, and signs of vulvovaginal atrophy. Menopause. 2014;21:450-8.

6. Roy S, Caillouette JC, Roy T, Faden JS. Vaginal pH is similar to follicle-stimulating hormone for menopause diagnosis. Am J Obstet Gynecol. 2004;190:1272-7.
7. The North American Menopause Society (NAMS). The 2020 genitourinary syndrome of menopause position statement of The North American Menopause Society. Menopause. 2020;27:976-92.

8. Palacios S, Cancelo MJ. Clinical update on the use of ospemifene in the treatment of severe symptomatic vulvar and vaginal atrophy. Int J Womens Health. 2016;8:617-26.

9. Rahn DD, Carberry C, Sanses TV, Mamik MM, Ward RM, Meriwether KV, et al. Vaginal estrogen for genitourinary syndrome of menopause: a systematic review. Obstet Gynecol. 2014;124:1147-56.

10. Suckling J, Lethaby A, Kennedy R. Local oestrogen for vaginal atrophy in postmenopausal women. Cochrane Database Syst Rev. 2006;(4):CD001500. Update in: Cochrane Database Syst Rev. 2016;(8):CD001500.

11. Martini P, Mazzatenta C, Saponati G. Efficacy and tolerability of fitostimoline in two different forms (soaked gauzes and cream) and citrizan gel in the topical treatment of second-degree superficial cutaneous burns. Dermatol Res Pract. 2011;2011:978291.

12. Farinella Z, Morale MC, Agosta MA, Rizza V. Stimulation of cell division in mouse fibroblast line 3 T3 by an extract derived from Triticum vulgare. Int J Tissue React. 1986;8:337-42.

13. Vanden Berghe DA, Yang QR, Totté J, Vlietinck AJ. Specific stimulation of human endothelial cells by Triticum vulgare extract and its biologically active fraction. Phytother Res. 1993;7:172-8.

14. Sanguigno L, Minale M, Vannini E, Arato G, Riccio R, Casapullo A, et al. Oligosaccharidic fractions derived from Triticum vulgare extract accelerate tissutal repairing processes in in vitro and in vivo models of skin lesions. J Ethnopharmacol. 2015;159:198-208.

15. Espitia De La Hoz FJ. Tratamiento no hormonal del síndrome genitourinario de la menopausia. Arch Med (Manizales). 2021;21(2). https://doi. org/10.30554/archmed.21.2.3996.2021

16. Gandhi J, Chen A, Dagur G, Suh Y, Smith N, Cali B, et al. Genitourinary syndrome of menopause: an overview of clinical manifestations, pathophysiology, etiology, evaluation, and management. Am J Obstet Gynecol. 2020;215:704-11.

17. Palacios S, Nappi RE, Bruyniks N, Particco M, Panay N; EVES Study Investigators. The European Vulvovaginal Epidemiological Survey (EVES): prevalence, symptoms and impact of vulvovaginal atrophy of menopause. Climacteric. 2018;21:286-91.

18. Nilsson K, Risberg B, Heimer G. The vaginal epithelium in the postmenopause - cytology, histology and $\mathrm{pH}$ as methods of assessment. Maturitas. 1995;21:51-6.

19. Hess R, Austin RM, Dillon S, Chang CC, Ness RB. Vaginal maturation index self-sample collection in mid-life women: acceptability and correlation with physician-collected samples. Menopause. 2008;15(4 Pt 1):726-9.

20. McEndree B. Clinical application of the vaginal maturation index. Nurse Pract. 1999;24:48, 51-2, 55-6.

21. Wiegel M, Meston C, Rosen R. The female sexual function index (FSFI) cross-validation and development of clinical cutoff scores. J Sex Marital Ther. 2005;31:1-20.

22. Meston CM, Freihart BK, Handy AB, Kilimnik CD, Rosen RC. Scoring and interpretation of the FSFI: what can be learned from 20 years of use? $\mathrm{J}$ Sex Med. 2020;17:17-25.

23. Espitia-De La Hoz FJ. Prevalence and characterisation of sexual dysfunctions in women, in 12 Colombian cities, 2009-2016. Rev Colomb Obstet Ginecol. 2018;69:9-21.

24. World Medical Association (WMA). WMA Declaration of Helsinki - Ethical principles for medical research involving human subjects. Fortaleza: 64 $4^{\text {th }}$ WMA General Assembly; 2013.

25. Colombia. Ministerio de Salud. Resolución 8430 de 1993 (octubre 4): Por la cual se establecen las normas científicas, técnicas y administrativas para la investigación en salud. Bogotá D.C.; 1993.

26. Chaturvedi A, Meswani R, Shah S, Deasi S, Lele J, Mehta P, et al. An Indian clinical trial to assess wound healing activity and safety of Fitostimoline $15 \%+1 \%$ cream as topical treatment of different type of wounds. J Plastic Dermatol. 2010;6:3.

27. Serafini G, Saponati G. Activity and tolerability of extracts of Triticum vulgare in diseases inflammatory-dystrophic. Dental Clinics. 2012;3:35-9.

28. Mollica G, Bonaccorsi G, Martinello R, Minale DG. Evaluation of efficacy and tolerability of fitostimoline vaginal cream (damor farmaceutici) in the treatment of vaginal inflammation and vulvar dystrophy: a double-blind randomized controlled trial. Gazzetta Medica Italiana Archivio per le Scienze Mediche. 2008;167:87-95.

29. Souza D, Machado T, Zoppa A, Cruz R, Garague A, Silva L. Ensaio da aplicacao de creme a base de Triticum vulgare na cicatrizacao de feridas cutaneas induzidas em equinos. Rev Bras Plantas Med. 2006;8:9-13.

30. Boscia F, Gala R, Pannaccione A, Secondo A, Scorziello A, Di Renzo G, et al. NCX1 expression and functional activity increase in microglia invading the infarct core. Stroke. 2009;40:3608-17.

31. Romanelli M, Macchia M, Panduri S, Paggi B, Saponati G, Dini V. Clinical evaluation of the efficacy and safety of a medical device in various forms containing Triticum vulgare for the treatment of venous leg ulcers — a randomized pilot study. Drug Des Devel Ther. 2015;9:2787-92.

32. Espitia De La Hoz FJ. Efectividad y seguridad del policresuleno en el tratamiento del síndrome genitourinario de la menopausia. Estudio controlado y aleatorizado. Arch Med (Manizales). 2020;20:282-94.

33. Panay N. Genitourinary syndrome of the menopause - dawn of a new era? Climacteric. 2015;18 (Suppl 1):13-7. 